\title{
Development of minimum toe clearance detector for a tripping prediction
}

\author{
Daiki Hamada ${ }^{\mathrm{a}}$, Shota Nakashima ${ }^{\mathrm{b}}$, Tomohiro Morio ${ }^{\mathrm{b}}$, Tatsuya Sugimura ${ }^{\mathrm{b}}$, Kazuo Haruyama ${ }^{\mathrm{a}}{ }^{*}$ \\ ${ }^{a}$ National Institute of Technology Ube College, 2-14-1 Tokiwadai, Ube, Yamaguchi, 755-8555, Japan \\ bYamaguchi University, 2-16-1 Tokiwadai, Ube, Yamaguchi, 755-8611, Japan \\ *Corresponding Author: haruyama@ube-k.ac.jp
}

\begin{abstract}
Falls of elderly during walking cause serious injury as fracture probably. Most of falls results from tripping, Minimum Toe Clearance(MinTC) indicating easiness to tripping was studied by a lot of researchers. It was reported that variability of MinTC was greater in the elderly than the young. This paper proposes a method to make a judgement on easiness to tripping based on the height of MinTC using One Dimensional Brightness Distribution Sensor. For the purpose, we detected the difference between normal gait and dangerous gait using our method. As a result, our method obtained the significant difference between normal and dangerous gait. In conclusion, we clearly judged normal gait or dangerous gait using our proposal system. In the future, we should test at the actual environment, develop further.
\end{abstract}

Keywords: fall, elderly, gait, Obrid-Sensor, Minimum Toe Clearance.

\section{Introduction}

Prediction of falls is the major problem. Each year approximately $20 \%$ of Japanese aged over 65 years causes a fall more than once ${ }^{(1)}$. Experience of fall make a feeling resistance for them in daily life and reducing their activity of daily living like moving and changing cloth. As the result, elders are certified the bedridden and need of nursing care. Nowadays, about 646000 of elders die due to the fall, fall is the second leading cause of unintentional injury death, after road traffic injuries ${ }^{(2)}$.

Recently years, about fall, major factor is tripping during walking ${ }^{(3)}$ and to occurs indoors ${ }^{(4)}$ were known. Especially, many elderly fall in living area such as living room and bed room ${ }^{(4)}$. Because elderly spend a long time in living area. A lot of factors which disperse elderly's attention were been in living area, elderly often move while doing something else, elderly fall as a result. Therefore, to judge they will be likely tripping, to acquire data on walking in living room is considered effective for prediction of falls.

As regards acquiring data on walking, major methods have been as using the motion tracking system ${ }^{(5)}$, an acceleration sensor and an inertial sensor ${ }^{(6)}$. However, motion tracking system is too large to use and acceleration sensor is troublesome to mount. So these method is not suit continuous acquiring data in daily life.

In this study, we focused on the Toe clearance, the height between toe and ground during walking. We proposed a system to make a judgement on easiness to tripping using One Dimensional Brightness Distribution Sensor without contact and restraint.

\section{Method}

\subsection{Toe Clearance}

During walking, it is known that each leg has two phase such swing phase and stance phase ${ }^{(7)}$. Swing phase is between toe off from the ground and heel on to the ground. The other term of walking is stance phase. Tripping is defined the touching ground during swing phase. For example, Fig. 1 indicated the changing phase of left leg seen from right side.

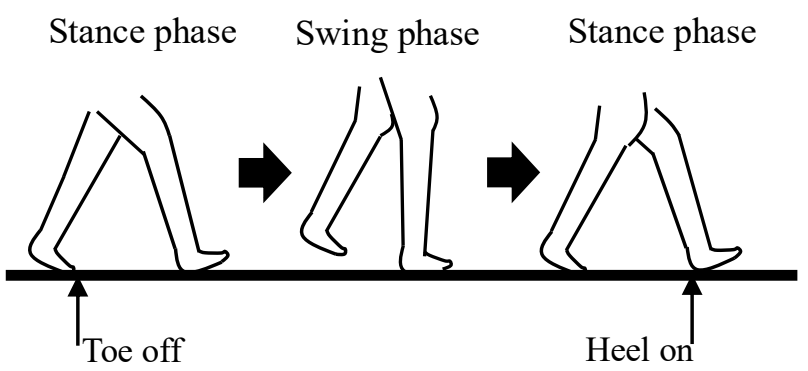

Fig. 1. Changing phase of left leg 


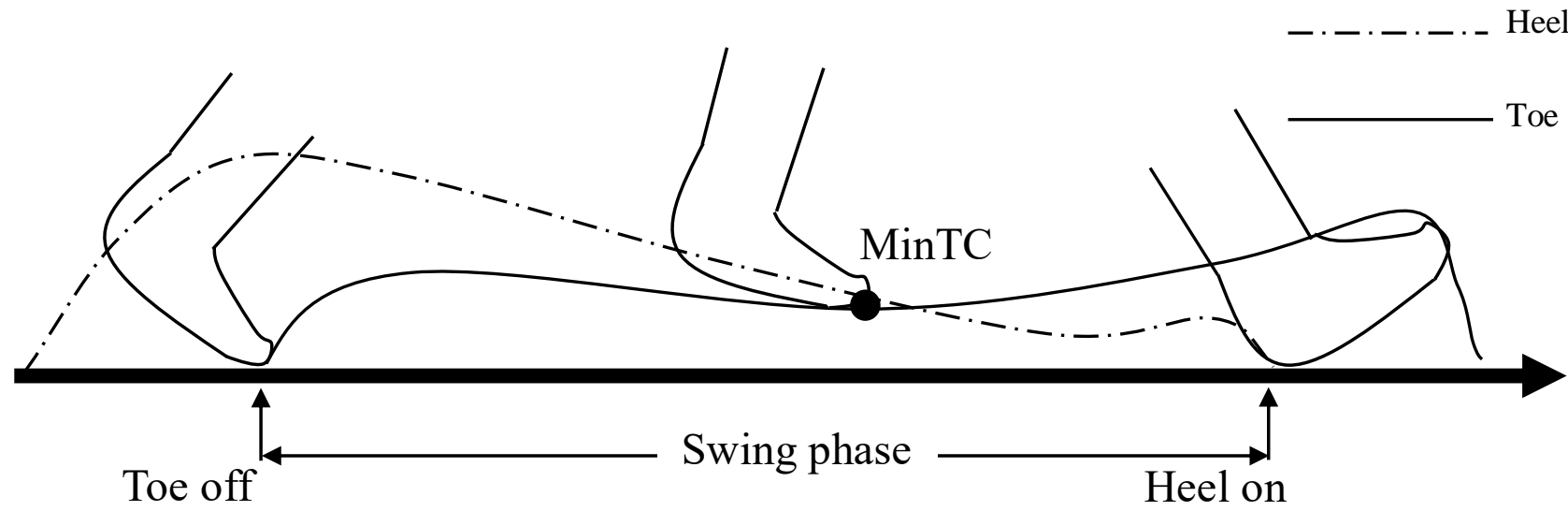

Fig. 2. Trajectories of heel and toe ${ }^{(8)}$

Toe Clearance (TC) is the height between toe and ground during walking. Between heel and ground is called Heel Clearance(HC). Fig. 2 indicates the trajectory of TC and HC during the swing phase ${ }^{(8)}$. The lowest TC is especially called Minimum Toe Clearance (MinTC), MinTC have been considered an important index of easiness to tripping. Begg et al. reported MinTC is seen at the approximately half of the swing phase in healthy person ${ }^{(9)}$. David A Winter assumed the coordinate model based on the measured value, reported that TC increased $4.5 \mathrm{~mm}$ by each ankle angle increasing 1.4 $\operatorname{deg}^{(10)}$. Mufadal A. Moosabhoy et al. suggested coordinates of the toe using following equations ${ }^{(11)}$ :

$$
\begin{gathered}
\mathbf{x}_{\mathrm{T}}=\mathrm{x}_{\mathrm{H}}+\mathrm{L}_{1} \sin (\alpha)+\mathrm{L}_{2} \sin (\alpha-\beta) \\
+\mathrm{L}_{3} \sin (\alpha-\beta+\gamma+\varphi) \\
\mathrm{y}_{\mathrm{T}}=\mathrm{y}_{\mathrm{H}}-\mathrm{L}_{1} \cos (\alpha)-\mathrm{L}_{2} \cos (\alpha-\beta) \\
-\mathrm{L}_{3} \cos (\alpha-\beta+\gamma+\varphi)
\end{gathered}
$$

where $\mathrm{x}_{\mathrm{H}}$ and $\mathrm{y}_{\mathrm{H}}$ are the fore-aft and vertical coodinates of the hip joint center, respectively; $L_{1}$ the length of the thigh segment; $L_{2}$ the length of the shank segment; $L_{3}$ the ankletoe distance; $\alpha$ the hip flexion angle; $\beta$ the knee flexion angle; $\gamma$ the ankle dorsiflexion angle; and $\varphi$ the neutral ankle angle.

\subsection{Analysis of tripping}

Mills et al. reported that no differences in mean MinTC were detected between the young and elderly, however interquartile range, statistical variability, of Min TC was seen the significant difference between elderly and young people ${ }^{(12)}$. Therefore, it can be considered that TC has two pattern. One of trajectory is with high MinTC. The other hand is with small MinTC. It has been recently considered by other researchers that elderly fall over by tripping when toe touched to the ground in case of small MinTC(13). Therefore, it was assumed that to count the frequency of small MinTC trajectory can estimate easiness to tripping.
Range of mean MinTC was reported $10 \mathrm{~mm}$ to $24 \mathrm{~mm}$ by a lot of researchers ${ }^{(13)}$. Therefore, in this paper, we set the threshold on $10 \mathrm{~mm}$ which was worst value of reported. If MinTC was less than $10 \mathrm{~mm}$, the gait was judged the dangerous gait with small MinTC, the risk of fall was judged high.

\subsection{One-Dimensional Brightness Distribution Sensor}

In this paper, One-Dimensional Brightness Distribution Sensor(Obrid-Sensor) was used to analysis gait based on MinTC. Obrid-Sensor ${ }^{(14)}$ is one of the method of human shape detecting with privacy-preserving. The sensor was constructed a cylindrical lens and a line sensor. A cylindrical lens acts as convex lens dependently on the direction of light. A schematic diagram is show in Fig. 3. Figure 3(a), (b), (c) show its overall view, side view and top view of the sensor respectively. As we see in Fig. 3(b), the cylindrical lends acts as the flat panel, the vertical brightness irradiated from different position is input into the sensor. As we see in Fig. 3(c), the cylindrical lends acts as convex lens, the horizontal brightness irradiated from different position is focus to the sensor. Summarizing the statement above, as Fig. 3(a) shows, the light radiated from the object's $R, R^{+}, R^{-}$enters the $S_{R}$ point of the line sensor that radiated from $\mathrm{C}^{+}, \mathrm{C}, \mathrm{C}^{-}$enters the $\mathrm{S}_{\mathrm{C}}$ point, and $\mathrm{L}^{+}, \mathrm{L}, \mathrm{L}^{-}$enters the $\mathrm{S}_{\mathrm{L}}$ point. Therefore, the output from the line sensor means the horizontal distribution of the brightness from the different vertical point.

In order to obtain the specific parameter using the ObridSensor, we used the background subtraction. Figure 4 indicated the correspondence between camera image and brightness distribution. Three brightness distributions shows background brightness, object brightness and difference between back ground and object brightness. First, the sensor fetched the background brightness distribution when nobody 


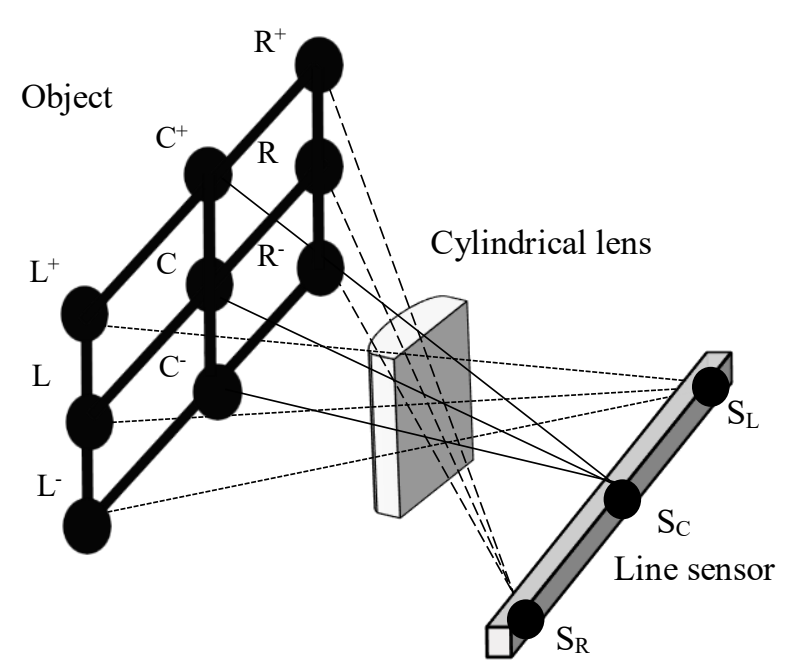

(a) Over view

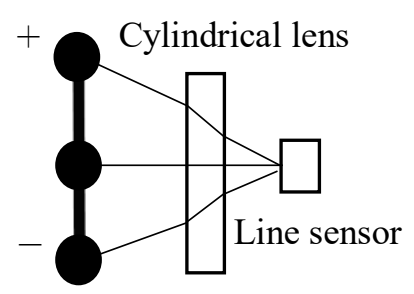

(b) Side view

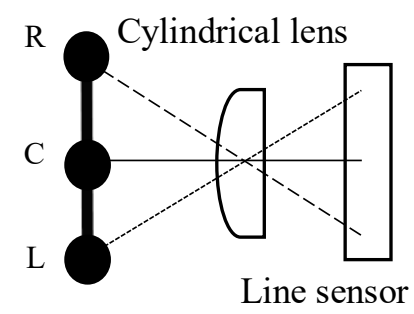

(c) Top view
Fig. 3. One-dimensional distribution sensor

was in. Next, the sensor fetched the current brightness distribution with person or object, the object brightness distribution was obtained. The absolute different from these brightness distributions can indicate the characteristic parameter. This characteristic parameter is estimated as OneDimensional distribution, means the position of the person or object. The peak value of brightness is changed by the color, shape and distance from the sensor.

\subsection{The method of finding the difference between normal and dangerous gait using Obrid-Sensor}

In this paper, it is necessary to extract the range of height less than $10 \mathrm{~mm}$. So next we state how to extract the horizontal brightness on threshold using Obrid-Sensor. We thought that combining with Obrid-Sensor, an infrared line laser, and an infrared transmit filter can extract only horizontal brightness. The infrared line laser can emit the laser as horizontal line. Sensor and infrared transmit filter were combined for detecting only the infrared light. To extract the horizontal brightness on threshold, we set the height the threshold both the infrared line laser and the sensor

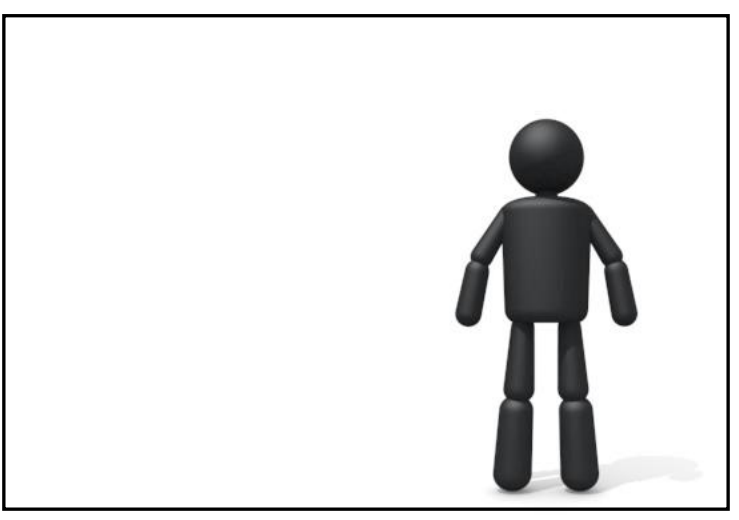

(a) Camera image

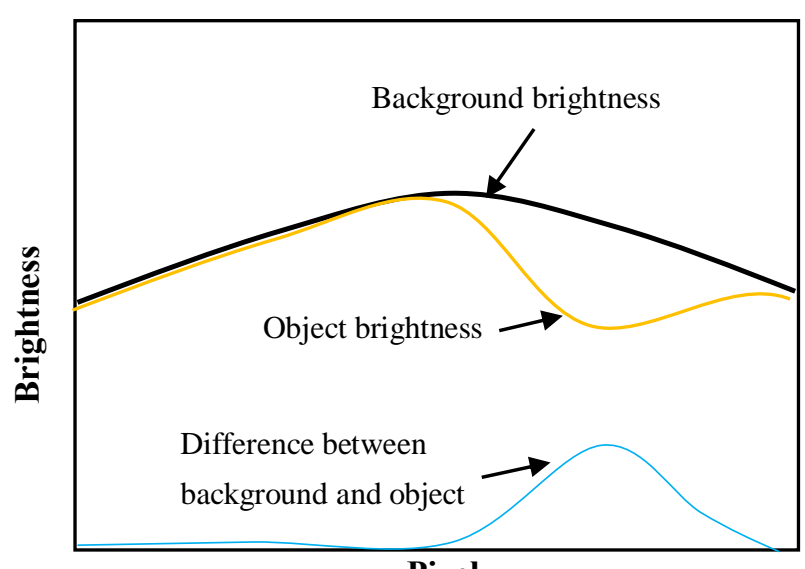

Pixel

(b) Brightness distribution

Fig. 4. Correspondence between camera image and brightness distribution

with filter. When a person entered the detection area, infrared light reflected and input into Obrid-sensor. This reflection light is the only horizontal component within threshold. At this time, it was available to extract the position using the background subtraction. In this study, the infrared reflected light is increased by distance from laser, the peak value of brightness is in inverse proportion to distance.

Next, we state the method of finding dangerous walking with small MinTC using the laser and the sensor. For instance, two trajectory pattern in Fig. 5 were considered, over and under the threshold around MinTC timing. Now, $\mathrm{t}_{1}$ defined the time before toe off, $t_{2}$ defined the time when floating foot around MinTC timing, $t_{3}$ defined the time after heel on. Brightness distributions on $t_{1}$ and $t_{3}$ were corresponding with the stance phase, two peaks based on detecting both foots were shown in these brightness distribution. A brightness distribution on $t_{2}$ was corresponding with the swing phase, single peak was shown in. If a foot is less than the threshold $10 \mathrm{~mm}$, the peak of brightness on $t_{2}$ was changed. This is due to detect a foot under threshold and reflect the infrared light from it. 

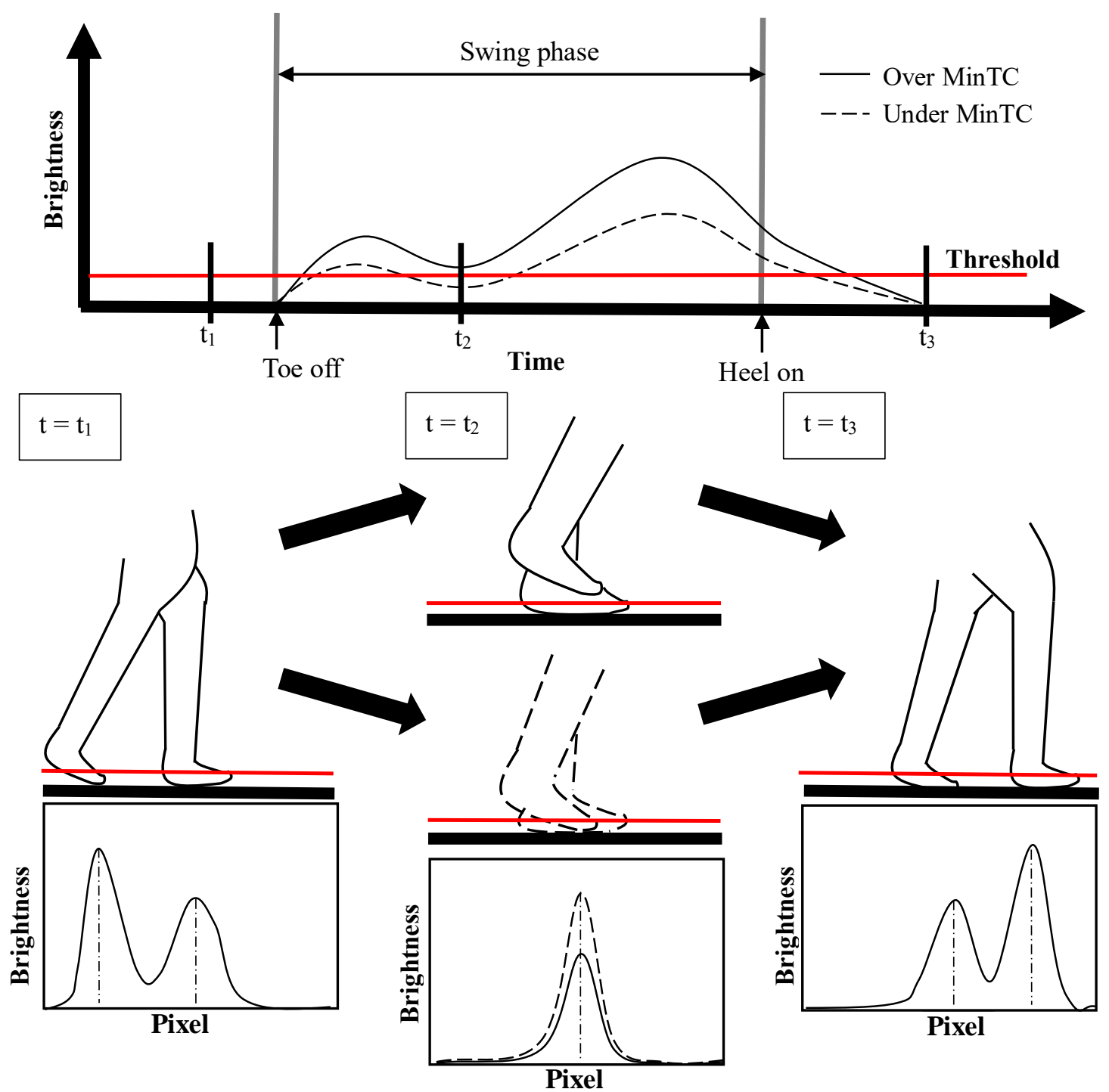

Fig. 5. Correspondence between foot state and brightness distribution

Therefore, the dangerous gait was judged when this changing peak of brightness was detecting.

\subsection{Judgement of the dangerous gait}

Next we suggested the method which judge normal gait or dangerous gait. The difference of brightness distribution between normal and dangerous gait in $\mathrm{t}_{2}$ can obtain as shown in 2.4. Now, we focused the size of brightness distribution, considered the time series of the size of brightness distribution. Figure 6 showed the ideal time series of size of brightness distribution. In this paper, the size of brightness distribution was acquired from sum of brightness distribution. The $x$ axis the time; the $y$ axis the sum of brightness; $h_{R}$ the sum of brightness when the right leg enters the detection area; $h_{L}$ the sum of brightness when the left leg enters. The $h_{R}$ is larger than $h_{L}$ because right leg is more near than left reg. First, the sum of brightness changes to $h_{R}$ when right leg enters the detection area. Next left leg enters, the sum of

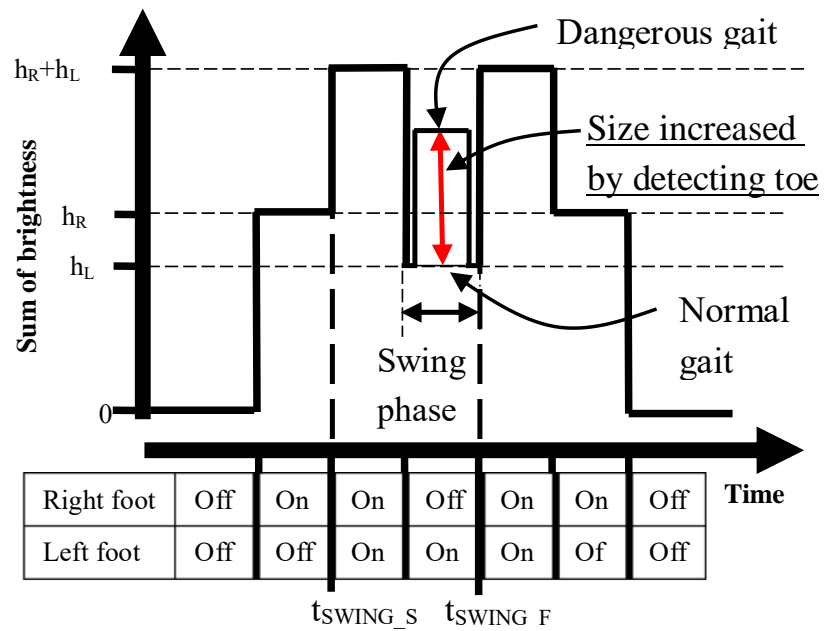

Fig. 6. Ideal time series of size of area

brightness changes to $h_{R}+h_{L}$. And next right leg exited, the sum of brightness changes to $h_{L}$. This timing was defined tswing_s. When right leg enters again, the sum of brightness to $h_{R}+h_{L}$. This timing was defined tswing F. Then, left leg 


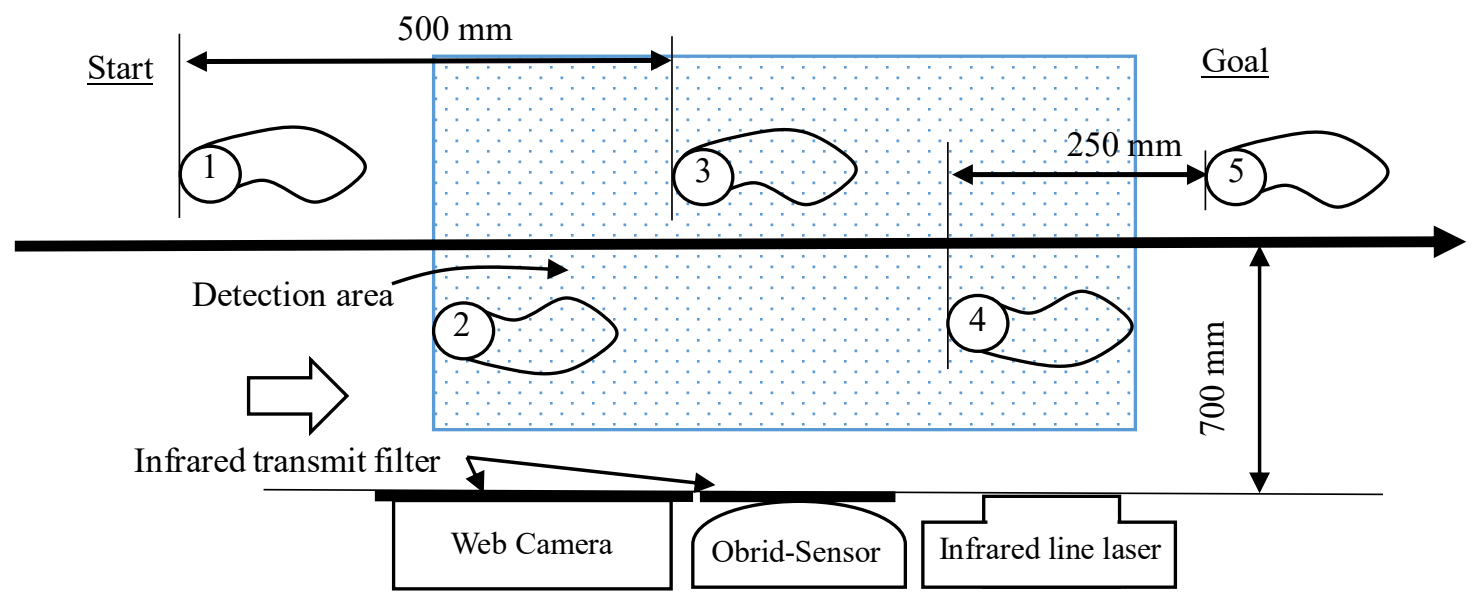

Fig. 7. Experiment environment

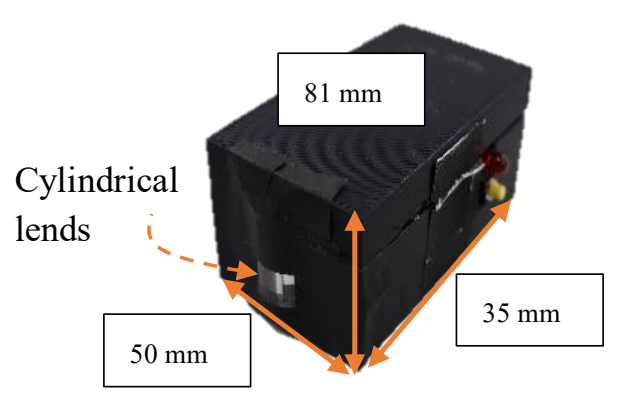

Fig. 8. One-dimensional brightness distribution Sensor exited, the sum of brightness changes to $h_{R}$. Finally, right leg exited, the sum of brightness changes to 0 . In this flow, the swing phase is between exiting right leg and entering again, from tswing s to tswing F. About normal walking, the flat value $h_{L}$ was obtained during swing phase because the sensor detects only left leg. However, about dangerous gait, the changing value with time was obtained during swing phase because the increasing size of area is caused by entering toe.

Next, the calculation way for judging is considered based on increasing sum of brightness. First, from tswiNG_s to $t_{\text {SwING_F }}$ was divided by $n, n$; the number of data between

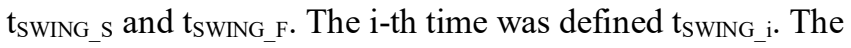
sum of brightness corresponding $t_{S W I N G} i$ is defined $\mathrm{S}\left(\mathrm{t}_{\mathrm{SWING}} \mathrm{i}\right)$, the $\mathrm{S}_{\text {mean }}$, the mean value from $\mathrm{t}_{\mathrm{SWING}} \mathrm{S}$ to $\mathrm{t}_{\mathrm{SWING}} \mathrm{F}$, was following equations:

$$
S_{\text {mean }}=\frac{1}{n} \sum_{i=0}^{n} s\left(t_{\text {SWING }_{-i}}\right)
$$

For instance, if the time series of the sum of brightness is like Fig. 6, $\mathrm{S}_{\text {mean }}$ should be seen the significant difference between normal gait and dangerous gait. In this paper, the judgement, normal gait or dangerous gait, was based on this significant difference.

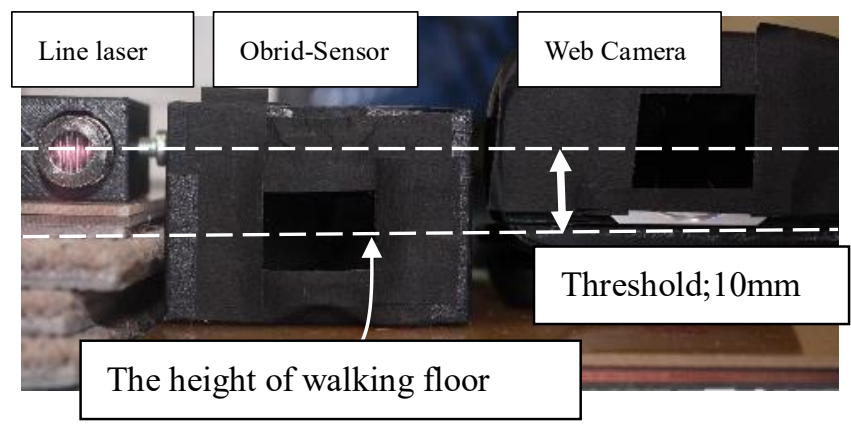

Fig. 9. Setting of sensor and laser height

\section{Experiment}

We should confirm the increasing brightness by detecting the foot under $10 \mathrm{~mm}$. Therefore, we did the walking trial and the time series data of the sum of distribution was collected both normal walking and dangerous walking using proposal method. Next, we estimated the swing phase roughly and compare the brightness distribution on normal gait to the brightness distribution on dangerous gait, we confirmed the detection of foot under $10 \mathrm{~mm}$. Finally, the significance difference based on 2.4, 2.5 was verified between normal and dangerous gait using the t-test. In all experiment, we took the video using web camera and used to confirm whether subjects came according to instructions.

\subsection{Experimental environment}

All subjects were 6 healthy males in twenties, the subject was informed of the purpose, contents, and influences on human body of the trial and got their acknowledgment before the trial. The experimental environment was set as shown in 


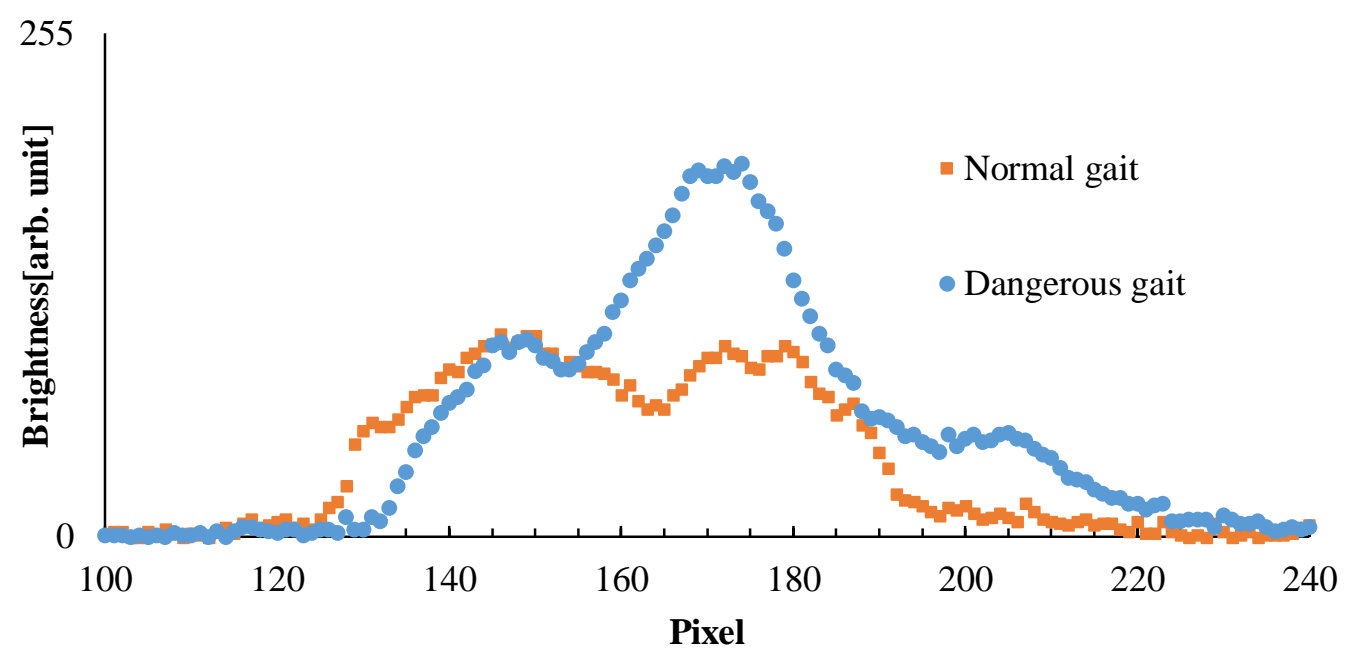

Fig. 10. Brightness distributions, normal and dangerous gait

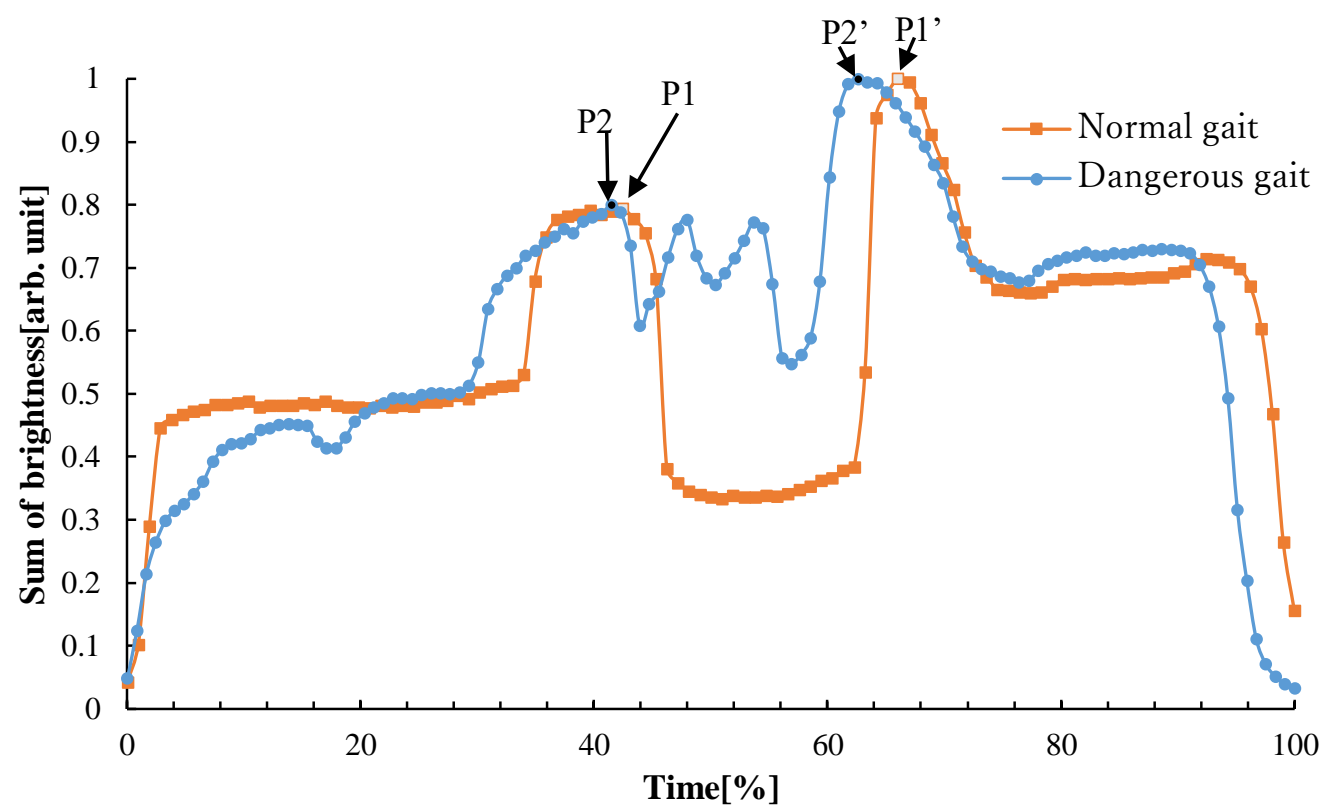

Fig. 11. Time series of sum of brightness, normal and dangerous gait

Fig. 7 with indoor light environment. The arrow in Fig. 7 indicates the traveling direction. First, we plot five markers to step on while gait. Markers was placed at equal intervals which the sensor could detect foots. Figure 8 were shown Obrid-Sensor using in the experiment. The focus length of cylindrical lens (N-SF11, Edmund optics, U.S.A) is $12.5 \mathrm{~mm}$, the lens width is $12.5 \mathrm{~mm}$ and the radius of curvature is 9.81 mm. A line sensor(TSL1402, ams, Austria) of Obrid-Sensor has the $256 \times 1$ pixel photo detectors, the width of detection of a line sensor is $18.3 \mathrm{~mm}$, processed by AVR microcomputer (ATmega328P, Atmel, U.S.A). An infrared laser (B075TVTV1M, summershine, China) was used in experiment, the center wave length is $808 \mathrm{~nm}$ and the power is $300 \mathrm{~mW}$. Figure 9 were shown the detection height setting. The interval between infrared line laser and the sensor was experimentally adjusted the best sensitivity point from preliminary experiments. The infrared transmit filter (IR80,
Fujifilm, Japan) was put on the lend of a Obrid-Sensor and a camera to cut the visible light. The subject was wearing socks while the experiment.

\subsection{Experimental method}

The subject walked in front of the sensor. For this trial, we decided the start and goal position as shown in Fig. 7. After the start sign, the subjects walked while stepping on the marker and the sensor started obtaining the brightness distribution until stepping on goal. The gait was instructed that subject's foot less than $10 \mathrm{~mm}$. Brightness distribution data were sampled at $20 \mathrm{~Hz}$. The walking speed was instructed preferably slow. This trial was made 5 times. After then, the gait was instructed that the subject's foot raised over $10 \mathrm{~mm}$, the subject walked 5 times again. In all walking, the speed was roughly set the same time using a stopwatch. 
Table 1. Result and verification between normal and dangerous gait

\begin{tabular}{|c|c|c|c|c|c|c|c|}
\hline & & \multicolumn{6}{|c|}{ Subjects } \\
\hline & & 1 & 2 & 3 & 4 & 5 & 6 \\
\hline$S_{\text {mean }}$ & Normal & $0.404 \pm 0.023$ & $0.338 \pm 0.009$ & $0.430 \pm 0.026$ & $0.552 \pm 0.042$ & $0.514 \pm 0.040$ & $0.382 \pm 0.041$ \\
\hline Mean & Dangerous & $0.564 \pm 0.091$ & $0.450 \pm 0.053$ & $0.535 \pm 0.035$ & $0.580 \pm 0.073$ & $0.674 \pm 0.077$ & $0.580 \pm 0.036$ \\
\hline \multicolumn{8}{|l|}{ \pm S. D. } \\
\hline \multicolumn{2}{|c|}{$P$ value } & $0.05>0.029$ & $0.05>0.009$ & $0.05>0.007$ & $0.05<0.398$ & $0.05>0.033$ & $0.05>0.001$ \\
\hline
\end{tabular}

\section{Result}

First, the increasing peak of brightness detecting a foot under $10 \mathrm{~mm}$, like Fig. 5, was confirmed each of results in all subjects. Figure 10 was shown two results of brightness distribution, normal and dangerous gait.

Next, the time series of sum of brightness was calculated each of a result. For instance, Fig. 11 was shown the time series of sum of brightness one of subject, normal and dangerous gait. The $\mathrm{x}$ axis and $\mathrm{y}$ axis were normalized to compare waveform. In normal gait, all result can estimate $t_{\text {SWING_S }}$ and $t_{\text {SWING_F }}$ shown 2.5. However, in dangerous gait, it is difficult to estimate $t_{\text {SWING_s }}$ and $t_{\text {SWING_F }}$ because the waveform was consecutive value. So the duration, which is from the timing of the local maximum sum of brightness caused detecting the both foots before swing phase $(\mathrm{P})$ to the timing of detecting the local maximum caused detecting the both foots after swing phase( $\left.\mathrm{P}^{\prime}\right)$, was used instead of $\mathrm{t}_{\text {SWING_S }}$

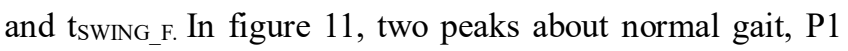
and $\mathrm{P} 1$ ', two peaks about dangerous gait, $\mathrm{P} 2$ and $\mathrm{P} 2$ ', are indicated. After then, $\mathrm{S}_{\text {mean }}$ was calculated each of result. Table 1 were shows the result of t-test, mean value and standard division of $\mathrm{S}_{\text {mean }}$. As a result, the significant difference was shown in 5 subjects.

\section{Discussion}

In Fig. 10, the large difference was confirmed between 155 pixel and 190 pixel. It was considered that the proposal system can detect right foot under threshold. A local maximum was shown both result around 145 pixel. It was the peak detected by the reflection light left foot same point.

In Fig. 11, we discuss about the waveform, a local maximum was confirmed around $40 \%$ both result. This was due to detect the both foot when left foot put on the floor. In normal gait, the flat value was shown from $45 \%$ to $63 \%$. In dangerous gait, the sum of brightness was sharply decreased and increased after a local maximum around $40 \%$. It was due to the reflecting brightness from a foot on swing phase was changing based on foot direction. Therefore, the large difference indicated 2.4 was shown by comparing the both waveform.

Table 1 were shown the significant difference based on $\mathrm{t}$ test was confirmed among 5 subject, therefore it is verified that the proposal method can judge whether dangerous gait or not. About a subject cannot confirmed significant difference, because this calculation was not suit to extract swing phase. In the future, the position of brightness peak should be combined the current method, so that it will be able to judge where foot influence the brightness. In addition, it should consider that influences of brightness from room or slipper.

Recently, the Laser Range Finder (LRF) was used for gait measurement. LRF could obtain the distance data of square area using laser. But the error of vertical detection width is $30 \mathrm{~mm}$ (HOKUYO, UAM-05LP-T301). Therefore, to obtain the characteristic value of MinTC using LRF is difficult because the reported mean MinTC is the range of 10-24 mm. On that point, the proposal method could obtain the characteristic value because the detection width was based on the line laser width. However, Ozawa proposed a gait measurement system using a $\operatorname{LRF}^{(15)}$. In this report, the effective detection range was reported $5.0 \mathrm{~m}$. This detection range was longer than our method because the effective detection range of our method was depending on line laser power. In the future, Expanding the effective range is essential for using at actual environment.

\section{Conclusions}

The results obtained show that despite their simplicity, this method give relatively accurate results and are sufficient for detecting the difference from normal walking to 
dangerous walking. Comparing the LRF, our method had an advantage as obtaining the characteristic value of MinTC. In the future, we should consider to expand the effective detection range.

\section{References}

(1) Takao Suzuki, Miho Sugiura, Taketo Furuna, Satoshi Nishikawa, Hideyo Yoshida, Tatsurou Ishizaki, Hunkyung Kim, Harumi Yukawa, and Hiroshi Shibata: "Association of Physical Performance and Falls Among the Community Elderly in Japan in a Five Year Followup Study", Journal of the Japan Geriatrics Society, Vol. 36, No. 7, pp. 472-478, 1999

(2) World Health Organization:"Fact sheets: Falls", http://www.who.int/news-room/fact-sheets/detail/falls, (refer on 16 January 2018)

(3) Mary E. Tinetti, Mark Speechley, and Sandra F. Ginter: "Risk Factors for Falls among Elderly Persons Living in the Community", The New England Journal of Medicine, Vol.319, No.26, pp.1701-1707,1988

(4) Siv Sadigh, Anne Reimers, Ragnar Anderson, and Lucie Laflamme: "Falls and fall-related injuries among the elderly: a survey of residential-case facilities in a Swedish municipality", Journal of Community Health, Vol. 29, No.2, pp.129-140, 2004

(5) Daniel T.H. Lai, Simon B. Taylor, Rezaul K. Begg: "Prediction of foot clearance parameters as a precursor to forecasting the risk of tripping and falling", Human Movement Science, No.31, pp.271-283, 2012

(6) Yasuaki OHTAKI, Koichi SAGAWA, Hikaru INOOKA : "A Method for the Gait Analysis in a Daily Living Environment by Body-Mounted Instruments", The Japan Society of Mechanical Engineers International Journal, Vol.44, No.4, pp.1125-1132, 2001

(7) Yoshihiro Ehara, Sumiko Yamamoto: "rinshohokoukeisokunyumon" (Introduction to clinical gait measurement), Ishiyaku publishers, 2008(in Japanese)

(8) Benoit Mariani, Stephane Rochat, Christophe J. Büla, and Kamiar Aminian: "Heel and Toe Clearance Estimation for Gait Analysis Using Wireless Inertial Sensors", IEEE Transactions on Biomedical Engineering, Vol. 59, No. 11, pp. 3162-3168, 2012

(9) Hanatsu Nagano, Rezaul K. Begg, William A. Sparrow, and Simon Taylor: "Ageing and limb dominance effects on foot-ground clearance during treadmill and overground walking”, Clinical Biomechanics, Vol.26,
No.9 pp.962-968, 2011

(10) Mufadal A. Moosabhoy, and Steven A. Gard: "Methodology for determining the sensitivity of swing leg toe clearance and leg length to swing leg joint angles during gait", Gait \& Posture, Vol.24, No.4, pp.493-501, 2005

(11)David A Winter: "Foot Trajectory in Human Gait: A precise and Multifactorial Motor Control Task", Physical Therapy, Volume 72, No.1, pp.45-53, 1992

(12) Mills PM, and Brarrett RS Morrison S: "Toe clearance variability during walking in young and elderly men", Gait \& Posture, No.28, Vol.1, pp.101-107, 2008

(13) Masayuki Soma: "Toe clearance and toe gripping strength in walking -a study for fall prevention-", Japanese Journal of Health Promotion and Physical Therapy, Vol.6, No.1, pp.1-7, 2016

(14) Shota Nakashima, Yuhki Kitazono, Kanya Tanaka, and Yuji Wakasa: "A Proposal of Method to Estimate Person's Upright and Falling Using the On-Dimensional Brightness Distribution Sensor", The Japanese Journal of the Institute of Industrial Applications Engineers, Vol.1, No.1, pp.20-26, 2013

(15) Mayumi Ozawa, Ayanori Yorozu, Tetsuya Matsumura, and Masaki Takahashi: "Gait Measurement System Using Laser Range Finder", Transactions of the Japan Society of Mechanical Engineers, Vol.79, No.801, pp.1550-1560, 2012 